\title{
An unusual cause of right lower abdominal pain in young female: A case report and review of literature
}

\author{
Priya Priyambada, Krishna Prasad Joshi,, Gohar Azhar \\ University of Arkansas for Medical Sciences, Little Rock, AR, USA
}

Received: December 22, 2015

Accepted: January 10, 2016

Online Published: January 25, 2016

DOI: $10.5430 /$ crim.v3n1p63

URL: http://dx.doi.org/10.5430/crim.v3n1p63

\begin{abstract}
Renal infarction is a serious medical emergency but its diagnosis is often delayed because it mimics many other conditions including renal stones, pyelonephritis, renal cancer and back pain. Early diagnosis as well as treatment is important for preventing recurrence of infarction and irreversible kidney damage. The most common causes of renal infarction are heart diseases leading to embolism, renal trauma and atheromatous disease. However, in some patients, the etiology remains unclear and is labeled as idiopathic renal infarction. We report an unusual case of right lower abdominal pain which was finally determined to idiopathic renal infarction after extensive investigation.
\end{abstract}

Key Words: Renal, infarction, Hypercoagulable, Antiphospholipid antibody

\section{INTRODUCTION}

Renal infarction is a very rare disease. ${ }^{[1]}$ The incidence of renal infarction was $1.4 \%$ in a study of 14,411 autopsies. ${ }^{[2]}$ Among 250,000 patients in Emergency Department, only $0.007 \%$ of total patients were diagnosed with acute renal infarction. ${ }^{[3]}$ The nonspecific clinical presentation, often similar to renal colic or pyelonephritis, is one of the main factors responsible for errors, with either a delay in diagnosis or misdiagnosis, resulting in a prolonged period of ischemia and irreversible renal damage.

The two most common causes of renal infarction are atrial fibrillation and renal artery injury. ${ }^{[4]}$ However, less frequent causes of renal infarction include hypercoagulable disorders, sickle cell disease, cocaine abuse, vasculitis, fibromuscular dysplasia and idiopathic renal infarction. ${ }^{[5-7]}$ We present an unusual case of idiopathic renal infarction in a young female.

\section{Case presentation}

A 29-year-old hispanic female presented to the Emergency Department with the complaint of right lower abdominal pain for 3 days. The pain was constant, sharp, and moderate to severe in intensity, aggravated by deep breathing with radiation to the right flank. She reported nausea, vomiting and diarrhea with red colored urine. She had an erythematous rash on her face one week ago but denied fever, increased urinary frequency or dysuria. She had no history of any chronic diseases including renal stones, heart disease, recurrent UTI, thromboembolic phenomenon or abdominal trauma. Last menstrual period was 2 weeks prior to presentation with obstetric history significant for one miscarriage and preeclampsia during second pregnancy. Family history was unremarkable. She had used oral contreaceptive pills in the past but was currently using an intra uterine device. She denied smoking, alcohol intake or use of recreational drugs. On examination, she was afebrile with normal vital signs. An

\footnotetext{
*Correspondence: Krishna Prasad Joshi; Email: krishnajoshi294@ gmail.com; Address: University of Arkansas for Medical Sciences, Little Rock, AR 72205, USA.
} 
erythematous malar rash was present on the face. Abdominal examination was normal except right costovertebral angle tenderness. Cardiovascular, respiratory, neuromuscular and genitourinary examinations were unremarkable.

From the clinical presentation, renal pathologies including renal stones, pyelonephritis and renal tumor were suspected. Complete blood count and basic metabolic panel were within normal limits. Liver function test showed mildly elevated bilirubin, aspartate aminotransferase and alanine aminotransferase. Lactate dehydrogenase was elevated to 584 with normal hepatitis serology. Urinalysis was positive for one plus proteinuria, 3-5 RBC/HPF and 6-10 WBC/HPF. Renal function showed blood urea nitrogen 10, Creatinine 0.8 , sodium 135, potassium 3.3, Chloride 102 with bicarbonate of 27. Urine pregnancy test was negative. Computerized Tomography (CT) of abdomen was reported as right upper pole pyelonephritis with multiple splenic cysts (see Figure 1). As the clinical picture and Urinalysis was not suggestive of pyelonephritis, Doppler ultrasound of the kidney was done that showed wedge shaped infarct within upper pole of the right kidney but no information about the renal arteries and aorta could be obtained.

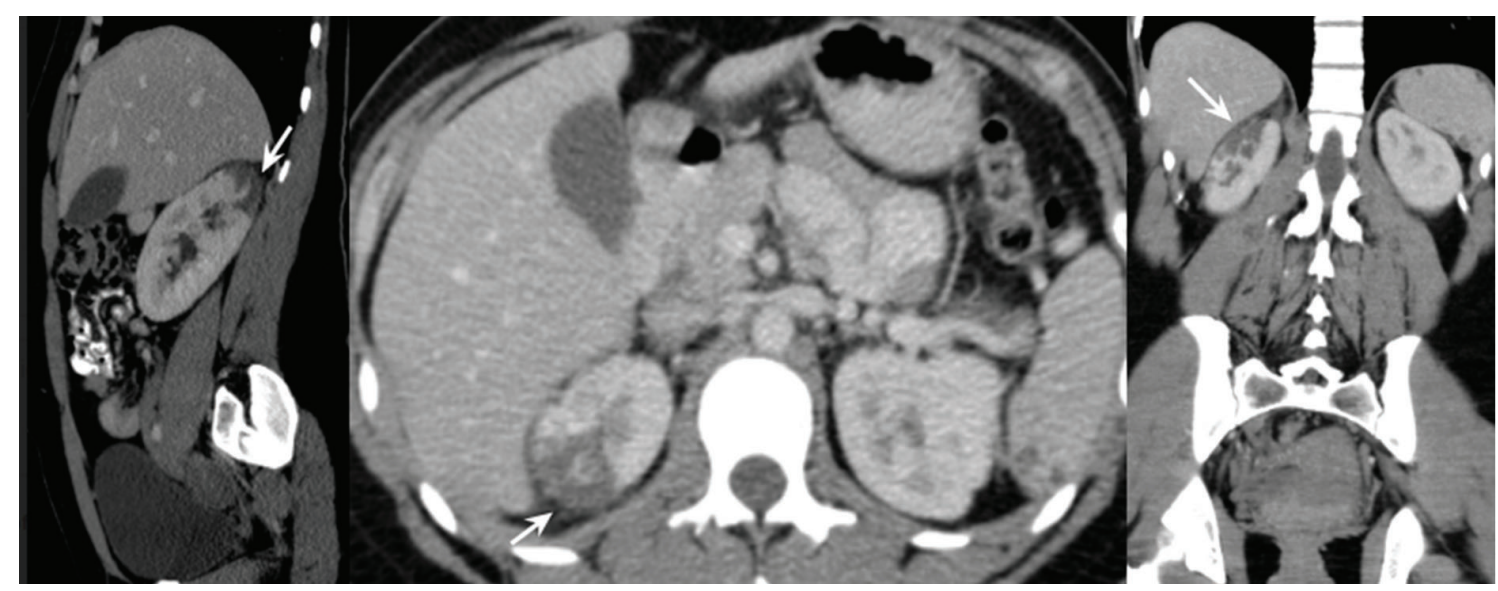

Figure 1. Computerized Tomography abdomen showing right kidney lesion

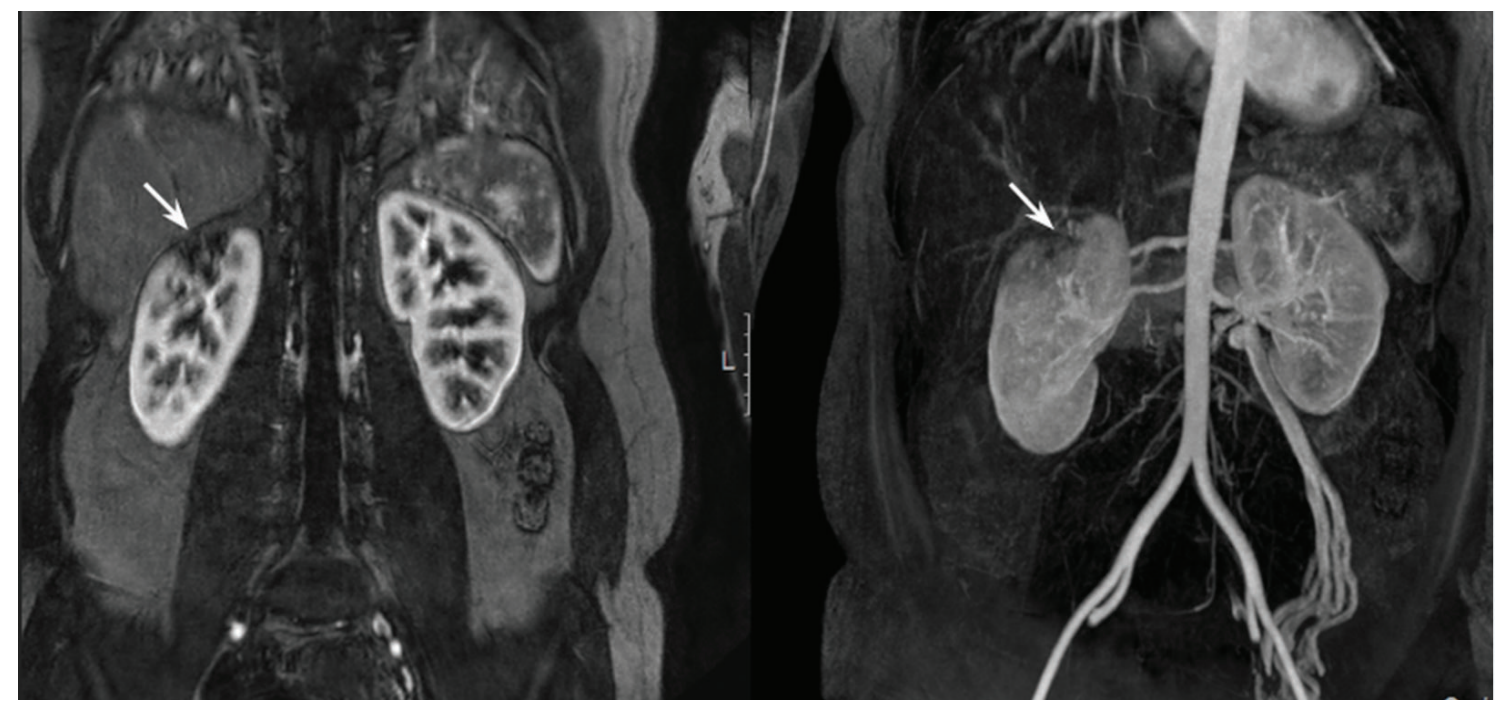

Figure 2. Magnetic resonance angiogram showing right renal infarct

As she continued to have right flank pain with hematuria, Magnetic Resonance Imaging (MRI) angiogram abdomen was done which showed wedge shaped hypo dense area in the upper pole of the right kidney representing a focal infarct and multiple splenic cysts (see Figure 2). Single right and two left renal arteries were identified with no evidence of fibromuscular dysplasia or renal vessel thrombosis. Initially, intravenous antibiotics were started for suspected pyelonephritis. In addi- 
tion, hypercoaguable work up was done with measurement of lupus anticoagulant, anticardiopipin antibody, beta glycoprotein, protein $\mathrm{S}$ and $\mathrm{C}$, factor $\mathrm{V}$ Leiden mutation and she was treated with Lovenox and Coumadin bridging for a potential thrombotic lesion causing the infarct. CT angiogram of the chest showed no cardiovascular or pulmonary lesions. Transthoracic echocardiogram was normal without any vegetation at valves. Antibiotics were discontinued after negative results of urine and blood cultures. Vasculitis screen was negative for antinuclear antibody, anti-double stranded DNA antibodies, and anti-neutrophil cytoplasmic antibodies.

Hypercoagulable panel showed normal beta glycoprotein, anticardiolipin antibody, protein $\mathrm{C}$ and $\mathrm{S}$. There was no evidence of factor $\mathrm{V}$ laden mutation. Procoagulant screening was positive for lupus anticoagulant. Her symptoms improved during hospital stay and she was discharged on Coumadin and scheduled to follow up in 12 weeks for repeat lupus anticoagulant. The repeat lupus anticoagulant test after 12 weeks was found to be negative and the probable diagnosis of antiphospholipid antibody syndrome was ruled out. The renal function which was normal at the beginning of the disease presentation was found to be normal with serum creatinine of 0.7 at 12 weeks follow up. The anticoagulation was discontinued and on continued follow up she had recovered fully.

\section{Discussion}

Renal infarction may present with the nonspecific clinical features and often overlaps with other diseases like nephrolithiasis or pyelonephritis. ${ }^{[5]}$ High index of suspicion with prompt work up is needed for timely treatment which is crucial in preventing the irreversible damage to kidney. Our patient presented with right lower abdominal pain which may be caused by the disease including urinary tract infection, appendicitis and ectopic pregnancy. Renal infarction may present with abdominal or lower back pain accompanied by nausea, vomiting and fever which may be seen with other causes of acute abdomen. Renal infarction can also alter rennin- angiotensin system leading to hypertension. ${ }^{[8]}$ Patients with renal infarction frequently present with microscopic hematuria but sometimes may even present with gross hematuria. ${ }^{[9]} \mathrm{Di}-$ agnosis of infarction might be obscured by the presence of elevated WBC, hematuria, pyuria, proteinuria which can mimic findings in other renal pathologies like renal stone, renal cell cancer that are more common. ${ }^{[10,11]}$ Renal stones and renal cell cancers are more common than renal infarction and are more likely to be detected by the initial radiological exams like CT or ultrasonography. But if the clinical pictures and lab findings do not correlate with imaging results, CT or MRI angiogram should be done to look for renal infarction.
CT scan abdomen in our case showed pyelonephritis in the beginning but clinical and lab findings were not suggestive of it which led to further evaluation with Ultrasound and MRI angiogram that revealed renal infarction with no pathology in the renal vessels. When renal infarct is suspected but is not visible in the early scans, repeat scans may be needed. ${ }^{[12]}$ In our case, renal infarction was not visible on CT scan initially but was seen on ultrasound done after 1 day and MRI done after 3 days of CT scan. Although CT scan of abdomen is investigation of choice in the diagnosis of renal infarction, ultrasound is valuable and cost effective alternative in pregnancy and in the cases when the clinical findings do not match with the diagnosis of CT scan.

Thromboembolic phenomena secondary to cardiovascular disease is the most common cause of renal infarction. The most common causes of thrombo-embolic phenomena are atrial fibrillation, myocardial infarction, bacterial endocarditis and rheumatic mitral stenosis. ${ }^{[13,14]}$ Hypercoagulable states like antiphospholipid syndrome and Polycythemia Vera are also major risk factors for renal infarction. ${ }^{[15]}$ In a large study of the patients with renal infarction, $25 \%$ were found to have emboli which originated from heart or aorta, 31\% had underlying renal artery injury secondary to fibro muscular dysplasia or spontaneous dissection and $16 \%$ were found to have congenital or acquired hypercoagulable disorder. However, in $29 \%$ of the patients the cause of renal infarction could not be determined after extensive evaluation and were classified as idiopathic renal infarct. ${ }^{[16]}$ In our patient, echocardiography and electrocardiogram were normal suggesting no structural heart disease or arrhythmia. Similarly, MRA did not show any renal artery injuries or fibromuscular dysplasia. Further work up was positive for lupus anticoagulant antibody but was negative for antinuclear antibody, anti-double stranded DNA antibodies, and anti-neutrophil cytoplasmic antibodies. Initially, the patient was suspected to have primary antiphospholipid antibody syndrome due to presence of lupus anticoagulant in the absence of preexisting underlying diseases like SLE. The diagnostic criteria for primary antiphospholipid syndrome require the persistent positivity of antiphospholipid antibody 12 weeks apart but in our case lupus anticoagulant was positive initially but was negative after 12 weeks. It should be noted that transient positive lupus anticoagulant can also be seen in infections and non-specific inflammations and might still mediate a hypercoagulable state. The possibility still remains that during the phase of the positive anti-phospholipid antibody, which might have existed before the patient presented to the ER, she had partial thrombosis of the renal artery which either dissolved spontaneously or soon after initiation of levonoxCoumadin therapy. However, because she did not satisfy 
the criteria for primary anti-phospholipid antibody syndrome and all other causes investigated for renal infarction were also negative, this case was categorized as the rare variety of idiopathic renal infarction.

\section{Conclusion}

During the evaluation of acute abdominal pain, renal infarction is often overlooked because it is an uncommon cause of abdominal pain. It may present with any type of abdominal pain and may not always be classical costovertebral angle pain. The patient in our case presented with lower abdominal pain radiating to right flank. Thorough evaluation for renal infarction in timely manner may prevent irreversible damage to kidney. When the suspicion of renal infarction is high like the history of recurrent pregnancy loss in young female or family history of hyper coagulable disorders and the initial radiological findings are not correlated with the clinical picture, contrast enhanced imaging like CT or MRI angiogram should be done for prompt diagnosis and timely management which may prevent irreversible damage.

\section{REFERENCES}

[1] Bourgault M, Grimbert P, Verret C, et al. Acute renal infarction: a case series. Clin J Am Soc Nephrol. 2013; 8: 392. PMid:23204242 http://dx.doi.org/10.2215/CJN.05570612

[2] Hoxie HJ, Coggin CB. Renal Infarction: Statistical study of two hundred and five cases and detailed report of an unusual case. Arch Intern Med. 1940; 65: 587. http://dx.doi.org/10.1001/arc hinte. 1940.00190090124007

[3] Paris B, Bobrie G, Rossignol P, et al. Blood pressure and renal outcomes in patients with kidney infarction and hypertension. J Hypertens. 2006; 24: 1649. Available from http://www.ncbi.nlm.nih.gov/pubmed/?term=J+Hypert ens. +2006+Aug\%3B24 (8)\%3A1649-54

[4] Antopolsky M, Simanovsky N, Stalnikowicz R, et al. Renal infarction in the ED: 10-year experience and review of the literature. Am J Emerg Med. 2012; 30: 1055. PMid:21871764 http: //dx.doi.org/10.1016/j.ajem.2011.06.041

[5] Javaid MM, Butt MA, Syed Y, et al. Acute renal infarction: an unusual cause of abdominal pain. Ren Fail. 2009; 31(5): 406-408. Available from: http://www.ncbi.nlm.nih.gov/pubmed/?ter $\mathrm{m}=$ Ren+Fail . $+2009 \% 3 \mathrm{~B}+31$ (5) $\% 3 \mathrm{~A} 406-408$

[6] Cabral RJ, Sousa L, Calaza C, et al. Acute segmental renal infarction due to factor V Leiden. Arch Esp Urol. 2009; 62(6): 486-488. Available from: http://www.ncbi.nlm.nih.gov/pubmed/?ter $\mathrm{m}=$ Arch + Esp+Urol $.+2009 \% 3 \mathrm{~B}+62(6) \% 3 \mathrm{~A} 486-488$

[7] Tsai SH, Chu SJ, Chen SJ, et al. Acute renal infarction: a 10year experience. Int J Clin Pract. 2007; 61(1): 62-67. Available from: http://www.ncbi.nlm.nih.gov/pubmed/?term= Int $+\mathrm{J}+\mathrm{Cl}$ in+Pract . $+2007 \% 3 \mathrm{~B}+61(1) \% 3 \mathrm{A62}-67$

[8] Lopez VM, Glauser J. A case of renal artery thrombosis with renal infarction. J Emerg Trauma Shock. 2010; 3(3): 302. PMid:20930986 http://dx.doi.org/10.4103/0974-2700.66569
[9] Korzets Z, Plotkin E, Bernheim J, et al. The clinical spectrum of acute renal infarction. Isr Med Assoc J. 2002; 4(10): 781-784. Available from: http://www.ncbi.nlm.nih.gov/pubmed/?ter $\mathrm{m}=\mathrm{Isr}+$ Med+Assoc+J . +2002+0ct\%3B4 (10)\%3A781-4

[10] Hazanov N, Somin M, Attali M, et al. Acute renal embolism: forty-four cases of renal infarction in patients with atrial fibrillation. Medicine. 2004; 83(5): 292-99. Available from: http://www.ncbi.nlm.nih.gov/pubmed/?term=Medi cine+(Baltimore) $.+2004+$ Sep $\% 3 B 83(5) \% 3 A 292-9$

[11] Domanovits H, Paulis M, Nikardjam M, et al. Acute renal infarction: clinical characteristics of 17 patients. Medicine. 1999; 78(6): 386-94. Available from: http://www.ncbi.nlm.nih.gov/pubmed/?ter $\mathrm{m}=$ Medicine+(Baltimore) $.+1999+$ Nov\%3B78 (6)\%3A386-94

[12] Kawashima A, Sandler CM, Ernst RD, et al. CT Evaluation of Renovascular Disease. Radio Graphics, 2000; 20: 1321-1340. http: //dx.doi.org/10.1148/radiographics.20.5.g00se141321

[13] Blum U, Billmann P, Krause T, et al. Effect of local low dose thrombolysis on clinical outcome in acute embolic renal artery occlusion. Radiology. 1993; 189(2): 549-554. PMid:8210388 http: //dx.doi.org/10.1148/radiology.189.2.8210388

[14] Golberg G. Renal infarction. Ann Emerg Med. 1985; 14(6): 611-614. Available from: http://www.ncbi.nlm.nih.gov/pubmed/?ter $\mathrm{m}=$ Ann+Emerg+Med .+1985+Jun\%3B14 (6)\%3A611-4

[15] Teplick JG, Yarrow MW. Arterial infarction of kidney. Ann Intern Med. 1955; 42(5): 691-700. Available from: http://www.ncbi.nlm.nih.gov/pubmed/14377238

[16] Bourgault M, Grimbert P, Verret $\mathrm{C}$, et al. Acute renal infarction: a case series. Clin J Am Soc Nephrol. 2013; 8: 392. PMid:23204242 http://dx.doi.org/10.2215/CJN.05570612 